Z. Klin. Chem. Klin. Biochem.

13. Jg. 1975 , S. $25-30$

\title{
Isolierung intakter Leberparenchymzellen durch eine modifizierte enzymatische Methode
}

\author{
Von H. Bojar ${ }^{1}$ ), K. Balzer, K. Reiners ${ }^{2}$ ), Marianne Basler, W. Reipen und W. Staib \\ Aus dem Institut für Physiologische Chemie II der Universität Düsseldorf
}

(Eingegangen am 10. Juni/16. Oktober 1974)

Herrn Prof. Dr. Karl Hinsberg zum 80. Geburtstag gewidmet

\begin{abstract}
Eine enzymatische Methode zur Isolierung intakter Parenchymzellen aus Rattenlebern mit einer Zellausbeute von 3-4 g Feuch tgewicht aus etwa $10 \mathrm{~g}$ schweren Lebern von 180-230 g schwcren Tieren wird beschrieben. Nach einer in-vitro-Vorperfusion mit einem calciumfreien Medium wurde Kollagenase $(200 \mathrm{mg} / \mathrm{l})$ und Calciumchlorid $(5,2 \mathrm{mmol} / \mathrm{l})$ hinzugegeben und die Leber $15 \mathrm{Minuten}$ bei $37^{\circ} \mathrm{C}$ perfundiert. Die mikromorphologische Unversehrtheit der Zelloberfläche wurde mit dem Rasterelektronenmikroskop nachgewiesen. Mit dieser Methode isolierte Leberparenchymzellen übertrafen hinsichtlich der Gluconeogenese- und Proteinsyntheseraten Leberschnitte und nach anderen Verfahren gewonnene Zellen. ATP/ADP- und Lactat/Pyruvat-Quotienten als Parameter für den Energie- bzw. Redoxstatus lagen mit 5,69 bzw. 8,64 im physiologischen Bereich. Die Integrität der membranständigen Hormonrezeptoren wurde durch den Nachweis der Sensibilität gegenüber Adrenalin, Glukagon und Insulin belegt. Die Glykogendeposition in Hepatocy ten gefasteter Ratten wurde durch Glukagon $(0,3 \mu \mathrm{mol} / 1)$ und Adrenalin $(1 \mu \mathrm{mol} / 1)$ um 84,9 bzw. $95,9 \%$ vermindert. Ähnlich wirkungsvoll stimulierten beide Hormone die Glykogenolyse in Parenchymzellen gefütterter Tiere. Die Harnstoffsynthese wurde durch Glukagon $(1 \mu \mathrm{mol} / \mathrm{l})$ um $29,5 \%$ stimuliert und durch Insulin $(10 \mathrm{nmol} / \mathrm{l})$ um 28,5\% gehemmt. Insulin unterdrückte in $10 \mathrm{nmolarer} \mathrm{Konzen}$ tration den Glukagoneffekt auf die Harnstoffbildung.
\end{abstract}

\section{Isolation of intact liver parenchymal cells by a modified enzymatic method}

An enzymatic method is described for isolating intact parenchymal cells from rat livers. 3-4 $\mathrm{g}$ cells (wet wcight) could be isolated from livers of rats weighing $180-230 \mathrm{~g}$. After an in vitro preperfusion of 15 minutes with a Ca-free buffer, collagenase (200 $\mathrm{mg} / \mathrm{l})$ and calcium chloride $(5.2 \mathrm{mmol} / \mathrm{l})$ were added. Perfusion was continued for another 15 minutes at $37^{\circ} \mathrm{C}$. Micromorphological integrity of cell membranes was demonstrated by scanning electron microscopy. With regard to rates of gluconeogenesis and protein synthesis, parenchymal cells isolated according to our method were found to be superior to liver slices and cells isolated by other methods. Ratios of ATP/ADP (5.69) and of lactate/pyruvate (8.64) as parameters of the energetic situation and the redox state resp. were found within the physiological range. Integrity of cell surface receptors was proved by their sensitivity to epinephrine, glucagon and insulin. Glucagon $(0.3 \mu \mathrm{mol} / \mathrm{l})$ and epinephrine $(1 \mu \mathrm{mol} / 1)$ reduced glycogen deposition in hepatocy tes of fasted rats by $84.9 \%$ and $95.9 \%$ resp. Both hormones stimulated glycogenolysis in parenchymal cells of fed rats to a similar extent. Urea synthesis was stimulated $29.5 \%$ by glucagon $(1 \mu \mathrm{mol} / 1)$, and inhibited $28.0 \%$ by insulin $(10 \mathrm{nmol} / 1)$. The stimulatory effect of glucagon $(1 \mu \mathrm{mol} / 1)$ was abolished by insulin $(10 \mathrm{nmol} / \mathrm{l})$ :

\section{Einleitung}

Die Interpretation von Stoffwechseluntersuchungen an der perfundierten Leber wird durch die Heterogenität der Zellpopulation erschwert. Darüber hinaus ist die Perfusionstechnik aufwendig und die Anzahl der gleichzeitig meßbaren Parameter begrenzt. Leberschnitte

\footnotetext{
1) Auszugsweise wurde diese Arbeit vorgetragen auf der gemeinsamen Tagung der Biochemischen Gesellschaften der Bundesrepublik Deutschland, der Schweiz und Österreichs in Innsbruck, 2.-5. Oktober 1973 und auf der Tagung der Deutschen Gesellschaft für Endokrinologie in Tübingen, 27. Februar-2. März 1974.

2) Die Arbeit beinhaltet wesentliche Teile der Dissertation von cand. med. $K$. Reiners (Med. Fak. der Universität Düsseldorf).
}

können in einer größeren Zahl aus einem Organ hergestellt werden und gestatten deshalb die gleichzeitige Untersuchung einer Reihe verschiedener Stoffwechselfunktionen. Die Nachteile dieses Modells liegen in einer starken Zellschädigung an den Schnittflächen, einer begrenzten Sauerstoff- und Substratdiffusion zu den tieferen Zellagen sowie in der Zellheterogenität. Mit geeigneten Methoden zur Isolierung und Fraktionierung vitaler Leberzellen lassen sich diese Probleme lösen. Mit unserem Isolationsverfahren können z.B. aus einer Rattenleber homogene Parenchymzellsuspensionen für bis zu hundert verschiedene gluconeogenetische Experimente gewonnen werden. Die Arbeit von Berry und Friend (1) war die Grundlage für die Entwicklung einer eigenen Methode zur Isolierung von Parenchymzellen. 


\section{Material und Methoden}

\section{Versuchstiere}

Es ivurden mit Standardkost ernährte männliche Wistar-llRatten (180-230 g) verwendet. Hungertiere hatten 22-24 Stunden vor Versuchsbeginn gefastet.

\section{Material}

Kollagenase (EC 3.4.24.3) CLS Typ II wurde von der Firma Worthington, Freehold (USA) bezogen, Hyaluronidase (EC 4.2.2.1) Typ I (bovine testes) von der Firma Sigma, St. Louis (USA). Adrenalin stammte von der Firma Hoechst, Frankfurt; kristallines Rinderinsulin wurde uns freundlicherweise von Herrn Prof. Dr. Brandenburg, Aachen und synthetisches Glukagon von der Firma Hoechst, Frankfurt zur Verfügung gestellt. Pentex-Rinderalbumin (Fraktion V) der Firma Miles-Seravac, Maidenhead (England) wurde vor Gebrauch entfettet und dialysiert. [U-14 C]L-Leucin bezogen wir von der Firma Amersham Buchler, Braunschweig, alle übrigen Chemikalien (Reinheitsgrad p.a.) von der Firma Merck, Darmstadt. Substrate wurden, wenn nicht anders angegeben, mit Testkombinationen der Firma Boehringer, Mannheim bestimmt. Das für einige Untersuchungen eingesetzte Aminosäuren-Gemisch enthielt mit Ausnahme des Leucins den Aminosäuren-Anteil des Medium 199 (2).

\section{Leberschnitte}

Leberschnitte wurden von einer in situ mit $50 \mathrm{ml}$ eiskaltem Krebs-Ringer-Phosphat-Puffer (pH 7,4) entbluteten Leber mit einem Mikrotom nach Stadie \& Riggs (3) angefertigt. Die Schnitte wogen 60-90 mg und waren 300-350 $\mu \mathrm{m}$ dick.

\section{Leberzellen}

Die von Howard et al. (4) angegebene Methode zur Isolierung von Leberzellen wurde ohne Modifikation übernommen.

Bei der von Berry (1) beschriebenen Methode wurde die in situ Perfusion durch in vitro Perfusion ersetzt.

Auf dieser Grundlage entwickelten wir das folgende Verfahren. Durch eine nicht rezirkulierende Vorperfusion in situ bei Raumtemperatur mit $150 \mathrm{ml}$ calciumfreiem Krebs-Ringer-BicarbonatPuffer $(\mathrm{pH} 7,4)$ wurde die Leber blutfrei gespült. Die Vorperfusion erfolgte mit einem Flow von $50-60 \mathrm{ml} / \mathrm{min}$. AnschlieBend wurde das Organ entnommen und bei $37^{\circ} \mathrm{C}$ in vitro 15 Minuten rezirkulierend mit $100 \mathrm{ml}$ calciumfreiem Krebs-RingerBicarbonat-Puffer $(\mathrm{pH} 7,4)$ bei einem Minutenvolumen von $50-60 \mathrm{ml}$ perfundiert. Dem Perfusat wurde dann $20 \mathrm{mg}$ Kollagenase, gelöst in $5 \mathrm{ml}$ einer $12,2 \mathrm{~g} / 1$ Calciumchloridlösung zugegeben (Endkonzentration im Perfusat $5,2 \mathrm{mmol} / \mathrm{l}$ ). Nach weiteren 15 Minuten wurde das Organ in $30 \mathrm{ml}$ calciumfreiem

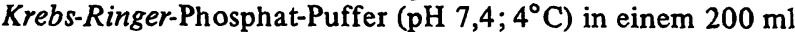
Polyäthylenbecher aufgenommen, indem die Leberlappen mehrfach eingeschnitten und die Zellen durch vorsich tiges Schütteln innerhalb von 2 Minuten suspendiert wurden. Gleichzeitig wurde die Suspension mit Carbogen $\left(5 \mathrm{Vol} \% \mathrm{CO}_{2}, 95 \mathrm{Vol} \% \mathrm{O}_{2}\right.$ ) durchperlt. Die Zellsuspension wurde durch ein Nylongewebe von $150 \mu \mathrm{m}$ Maschenweite filtriert und auf ein Volumen von $80 \mathrm{ml}$ aufgefüllt. Durch Zentrifugation $\left(4^{\circ} \mathrm{C}, 1 \mathrm{~min}, 500 \mathrm{U} / \mathrm{min}\right.$, Sorvall RC2-B, Rotor SS 34) trennten wir die Parenchymzellen von Zelldetritus und Nichtparenchymzellen. Der Waschvorgang wurde zweimal mit $80 \mathrm{ml}$ Krebs-Ringer-PhosphatPuffer $(\mathrm{pH} \mathrm{7,4)}$ wiederholt. Nach dem letzten Waschen wurde das Zellpellet gewogen und mit Krebs-Ringer-Bicarbonat-Puffer $(\mathrm{pH} 7,4)$ eine Zellsuspension hergestellt, von der wir aliquote Mengen den Inkubationsansätzen zugaben. Wenn nicht anders angegeben, wurden die Ansätze unter Carbogenatmosphäre $\left(5 \mathrm{Vol} \% \mathrm{CO}_{2}, 95 \mathrm{Vol} \% \mathrm{O}_{2}\right.$ ) in Polyäthylenröhrchen (Szintillationsgefäße der Firma Packard Instrument, Frankfurt) in einem Schüttelbad inkubiert. Die Temperatur betrug $37^{\circ} \mathrm{C}$, die Schüttelfrequenz $110-120 / \mathrm{min}$, die Amplitude $2,5 \mathrm{~cm}$.

\section{ATP, ADP, Lactat, Py ruvat}

$600 \mathrm{mg}$ Zellen wurden in $5 \mathrm{ml}$ Krebs-Ringer-Bicarbonat-Puffer . $(\mathrm{pH} 7,4) \mathrm{mit} 5,5 \mathrm{mmol} / 1$ Glucose, $20 \mathrm{~g} / 1$ Albumin und $0,606 \mathrm{~g} / \mathrm{l}$ des Aminosäuren-Gemisches inkubiert. Nach $30 \mathrm{bzw} .60 \mathrm{~min}$ beendeten wir die Inkubation durch Zusatz von $1 \mathrm{ml} 300 \mathrm{~g} / \mathrm{kg}$ $\mathrm{HClO}_{4}$. Anschließend wurde unter Kühlung homogenisiert (30 s, $15000 \mathrm{U} / \mathrm{min}$, Ultra-Turrax der Firma Ika-Werk, Staufen), in einer Kühlzentrifuge $(10 \mathrm{~min}, 20000 \mathrm{U} / \mathrm{min}$, Sorvall RC 2B, Rotor SM 24) zentrifugiert und im Úberstand nach Abpuffernmit $50 \mathrm{~g} / 1 \mathrm{~K}_{2} \mathrm{CO}_{3}$-Lösung die angegebenen Substrate gemessen. Die Bestimmung des ATP erfolgte nach Lamprecht \& Trautschold (5).

\section{Gluconeogenese}

$60 \mathrm{mg}$ Zellen von Hungertieren wurden in $2 \mathrm{ml}$ Krebs-RingerBicarbonat-Puffer ( $\mathrm{pH} \mathrm{7,4)} \mathrm{mit} 10 \mathrm{mmol} / 1$ Pyruvat inkubiert. Nach 60 min stoppten wir die Reaktion mit $0,2 \mathrm{ml} 300 \mathrm{~g} / \mathrm{kg}$ $\mathrm{HClO}_{4}$ und zentrifugierten $10 \mathrm{~min}$ bei $3000 \mathrm{U} / \mathrm{min}$ (Zentrifuge: Heraeus Christ, Osterode, Typ UJ3). Die Gluconeogeneseraten der Leberschnitte ließen sich nach Inkubation eines Schnittes in $2 \mathrm{ml}$ des gleichen Puffers bestimmen. Nach 60 min wurde $0,5 \mathrm{ml} 100 \mathrm{~g} / 1$ Trichloressigsäure zugegeben und wie beschrieben zentrifugiert. Die Überstände setzten wir nach Abpuffern mit $\mathrm{KHCO}_{3}$ zur Bestimmung des Glucosegehaltes ein.

\section{Proteinsynthese}

$60 \mathrm{mg}$ Zellen bzw. Leberschnitte wurden in $2 \mathrm{ml}$ Krebs-RingerBicarbonat-Puffer (pH 7,4) mit 5,5 mmol/1 Glucose, 0,606 g/l Aminosäuren-Gemisch und $100 \mu \mathrm{l}$ Leucinlösung (entsprechend $0,2 \mu \mathrm{Ci}\left[\mathrm{U}^{-14} \mathrm{C}\right] L$-Leucin) $60 \mathrm{~min}$ inkubiert. Die Zugabe von $0,2 \mathrm{ml} 300 \mathrm{~g} / \mathrm{kg} \mathrm{HClO}_{4}$ beendete die Reaktion: Nach Zentrifugation (10 min, $3000 \mathrm{U} / \mathrm{min}$, Heraeus Christ, Typ UJ3) und drei Waschvorgängen mit $0,1 \mathrm{ml}$ gesättigter Leucinlösung und $3 \mathrm{ml} 60 \mathrm{~g} / \mathrm{kg} \mathrm{HClO}_{4}$ wurde das Sediment in $0,5 \mathrm{ml}$ Hyamine (Firma Packard, Frankfurt) aufgenommen. Je zweimal 0,2 ml davon wurden mit $10 \mathrm{ml}$ Bray'scher Lösung versetzt und die Radioaktivität im Tri-Carb-Scintillation-Spectrometer Typ 3380 der Firma Packard bestimmt.

\section{Glykogensy nthese}

In $3 \mathrm{ml}$ Krebs-Ringer-Bicarbonat-Puffer ( $\mathrm{pH} 7,4$ ), dem die angegebenen Substrate (Lactat $10 \mathrm{mmol} / 1$, Alanin $10 \mathrm{mmol} / 1$, Fructose $10 \mathrm{mmol} / 1 \mathrm{bzw}$. Glucose $30 \mathrm{mmol} / \mathrm{l}$ ohne oder mit Zusatz von 5,10 oder $15 \mathrm{mmol} / 1$ Alanin) zugegeben waren, wurden $100 \mathrm{mg}$ Zellen von Hungertieren $90 \mathrm{~min}$ inkubiert. Mit $9 \mathrm{ml}$ heißer $300 \mathrm{~g} / 1 \mathrm{KOH}$ wurde die Reaktion beendet. Die Bestimmung des Glykogens erfolgte nach Seifter et al. (6). Der Einfluß von Glukagon $(0,3 \mu \mathrm{mol} / \mathrm{l}) \mathrm{bzw}$. Adrenalin $(1 \mu \mathrm{mol} / \mathrm{l})$ wurde bei einer Substratkonzentration von $30 \mathrm{mmol} / 1$ Glucose und $5 \mathrm{mmol} / 1$ Alanin untersucht; dabei benuțten wir die Versuchsanordnung wie vorstehend beschrieben.

\section{Glykogenolyse}

$40 \mathrm{mg}$ Zellen wurden in $1,2 \mathrm{ml}$ Krebs-Ringer-Bicarbonat-Puffer $(\mathrm{pH} 7,4)$ mit Glukagon $(0,3 \mu \mathrm{mol} / \mathrm{l})$ bzw. Adrenalin $(1 \mu \mathrm{mol} / 1)$. $30 \mathrm{~min}$ inkubiert. Die Aufarbeitung und Glykogenbestimmung erfolgten wie zuvor beschrieben.

\section{Harnstoffsynthese}

$70 \mathrm{mg}$ Zellen wurden in $2 \mathrm{ml} \mathrm{Krebs-Ringer-Bicarbonat-Puffer}$ (pH 7,4) mit $10 \mathrm{mmol} / 1$ Alanin unter Zusatz von Glukagon $(1 \mu \mathrm{mol} / 1)$, Insulin $(10 \mathrm{nmol} / \mathrm{l}) \mathrm{bzw}$. Glukagon und Insulin $60 \mathrm{~min}$ inkubiert und anschließend $5 \mathrm{~min}$ bei $3000 \mathrm{U} / \mathrm{min}$ zentrifugiert (Sorvall RC2-B, Rotor SM 24). Im Überstand bestimmten wir den Harnstoffgehalt.

\section{Bestimmung des Zelltrocken- und Zellfeuch tgewichtes} Als Trockengewicht wurde das Gewicht des Trichloressigsäureunlöslichen Materials nach Trocknung bei $110^{\circ} \mathrm{C}$ bis zur Gewichtskonstanz bezeichnet. $1 \mathrm{~g}$ des locker gepackten feuchten Zellpellets, das wir nach dem Waschen entsprechend den Angaben im Abschnitt „Leberzellen“ erhielten, entsprach im Mittel einem Trockengewicht des Trichloressigsäure-unlösliçhen Materials von $78,6 \mathrm{mg}$.

Soweit die Ergebnisse auf das Zellfeuchtgewicht bezogen wurden, legten wir wegen des erheblichen Pufferanteils im obengenannten 
Zellpellet nicht dessen Gewicht zugrunde, sondern ermittelten das Feuch tgewicht der Zellen nach Zentrifugation (1 min, $3000 \mathrm{U} / \mathrm{min}$ Sorvall RC2-B, Rotor SM 24) von $1 \mathrm{ml}$ der für die Versuche eingesetzten Zellsuspension. $1 \mathrm{~g}$ der abzentrifugierten Zellen entsprach im Mittel $1,41 \times 10^{8}$ Zellen.

\section{Statistik}

Die statistische Auswertung erfolgte nach dem t-Test nach Student. Angegeben ist jeweils der Mittelwert \pm Standardabweichung des Mittelwertes.

\section{Rasterelektronenmikroskopie}

Für die rasterelektronenmikroskopischen Untersuchungen wurden die Zellen nach Rosenbauer \& Schlösser (7) präpariert und im Rasterelektronenmikroskop (JEOL-JSM-U3) bei einer Beschleunigungsspannung von $25 \mathrm{kV}$ photographiert. Für die Anfertigung der Aufnahmen sind wir Herrn Dr. H. W. Schlösser zu Dank verpflich tet.

\section{Ergebnisse}

Aus der etwa $10 \mathrm{~g}$ schweren Leber einer 180-230 g schweren Ratte ließen sich nach unserem Verfahren 3-4 g Parenchymzellen isolieren. Die Kontamination mit Nichtparenchymzellen betrug 3-5\%.

Die morphologische Analyse im Rasterelektronenmikroskop (Abb. 1) zeigte Parenchymzellen, die ihre polyedrische Gestalt verloren und sich abgerundet hatten. Die Zellen wiesen eine unregelmäßig granulierte Oberflächenstruktur auf; Membrandefekte waren nicht erkennbar.

Nach unserem Verfahren isolierte Zellen wurden verglichen mit Leberschnitten und mit Zellen, die nach den Methoden von Howard (4) und nach Berry (1) in unserem Laboratorium isoliert wurden. Dazu wurden die Gluconeogenese- und Proteinsyntheseraten herangezogen.

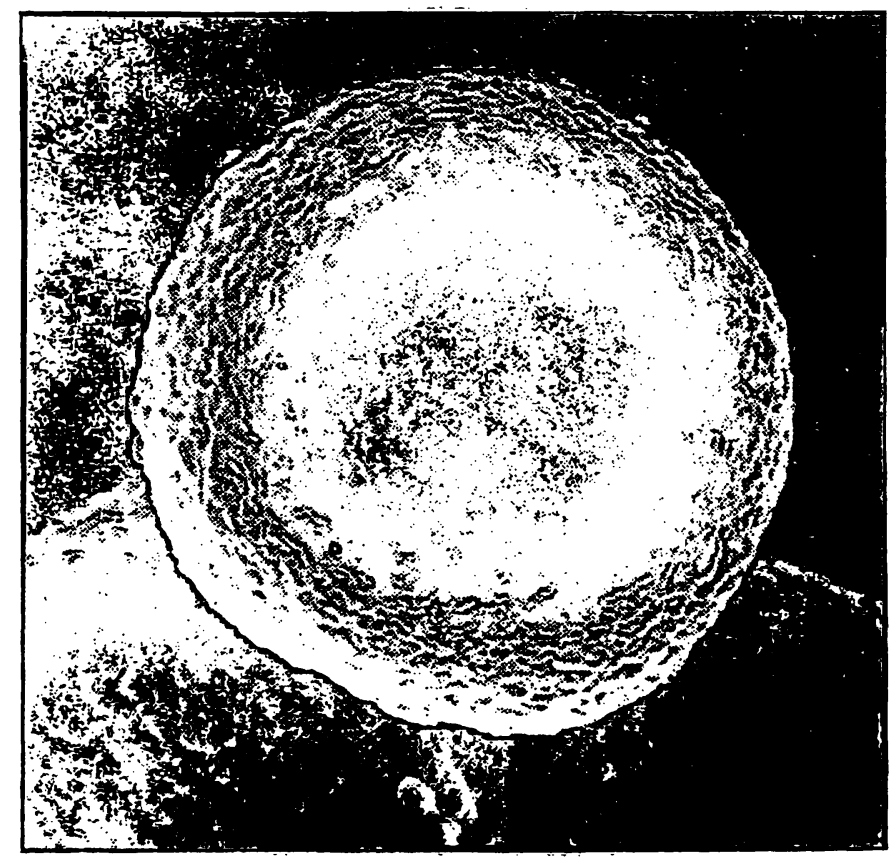

Abb. 1. Rasterelektronénmikroskopische Aufnahme isolierter Leberparenchymzellen (Vergrößerung etwa $2500 \mathrm{fach}$ ) Die Zellen haben ihre polyedrische Gestalt verloren und sich abgerundet. Sie weisen eine granulierte, teilweise flach gefurchte Oberfläche auf. Substanzdefekte der Zellmembran sind nicht erkennbar.
Die Gluconeogeneseraten aus $10 \mathrm{mmol} / 1$ Pyruvat waren bei Leberschnitten bzw. bei Zellen, die nach Howard (4) isoliert wurden, mit 65,84 $\pm 8,04(n=5)$ bzw. 65,51 $\pm 11,07 \mu \mathrm{mol}$ Glucose $/ \mathrm{h} \cdot \mathrm{g}$ Trockengewicht $(\mathrm{n}=7)$ etwa gleich hoch. Nach Berry (1) isolierte Zellen produzierten signifikant $(\mathrm{p}<0,05)$ größere Mengen Glucose $(113,42 \pm 11,88 \mu \mathrm{mol} / \mathrm{h} \cdot \mathrm{g}$ Trockengewicht; $\mathrm{n}=12)$. Mit 326,65 \pm 7,09 $\mu \mathrm{mol}$ Glucose/h $\cdot \mathrm{g}$ Trockengewicht $(n=8)$ wiesen die nach unserem Verfahren isolierten Zellen etwa fünf- bzw. dreifach höhere Gluconeogeneseraten auf.

Bei der Messung der Proteinsynthese aus einem Aminosäuren-Gemisch unter Zusatz von [U- ${ }^{14} \mathrm{C}$ ]-L-Leucin erzielten sämtliche isolierten Zellen signifikant $(p<0,05)$ höhere Werte als Leberschnitte. Während bei Schnitten Einbauraten von $0,81 \pm 0,10 \mu \mathrm{Ci} / \mathrm{h} \cdot \mathrm{g}$ Trockengewicht ( $n=4)$ gezählt wurden, erreichten nach Howard (4) isolierte Zellen $1,64 \pm 0,16 \mu \mathrm{Ci} / \mathrm{h} \cdot \mathrm{g}$ Trockengewicht $(\mathrm{n}=5)$. Nach Berry (1) isolierte Zellen wiesen mit $3,19 \pm 0,56 \mu \mathrm{Ci} / \mathrm{h} \cdot \mathrm{g}$ Trockengewicht $(\mathrm{n}=4)$ im Vergleich zu den Leberschnitten viermal höhere Einbauraten auf, nach unserem Verfahren isolierte Leberzellen mit $6,65 \pm 0,47 \mu \mathrm{Ci} / \mathrm{h} \cdot \mathrm{g}$ Trockengewicht $(\mathrm{n}=7)$ sogar die achtfachen Einbauraten von [U- $\left.{ }^{14} \mathrm{C}\right]-L$-Leucin.

Nachdem sich beim Vergleich der Gluconeogenese- und Proteinsyntheseraten die Überlegenheit unserer Isolationsmethode gezeigt hatte, wurden weitere Untersuchungen nur noch an den nach diesem Verfahren isolierten Zellen vorgenommen.

Zur Beurteilung des Redox- und Energiestatus wurden der Lactat/Pyruvat- bzw. der ATP/ADP-Quotient herangezogen. Der Lactat- und Pyruvatgehalt der Zellen wurde nach 30- und 60-minütiger Inkubation (siehe oben) bestimmt (jeweils $n=9)$. Nach 30 min ergab sich für Lactat ein Wert von $69,57 \pm 14,52$, für Pyruvat ein Wert von $9,83 \pm 2,21 \mu \mathrm{mol} / \mathrm{g}$ Trockengewicht. Die entsprechenden Zahlen beliefen sich nach $60 \mathrm{~min}$ auf $68,57 \pm 15,70$ bzw. 8,37 $\pm 2,1.4 \mu \mathrm{mol} / \mathrm{g}$ Trockengewicht. Der Mittelwert des Lactat/Pyruvat-Quotienten betrug nach $30 \mathrm{~min}$ $7,25 \pm 0,51$ und nach $60 \min 8,64 \pm 0,62$.

Der Gehalt der Zellen an $A T P$ und $A D P$ wurde nach einer Inkubation von 60 min bestimmt (jeweils $n=9$ ). Für ATP ergab sich ein Wert von $8,56 \pm 1,18$, für ADP ein Wert von $1,59 \pm 0,23 \mu \mathrm{mol} / \mathrm{g}$ Trockengewicht. Die ATP/ADP-Quotienten lagen im Mittel bei 5,69 0,66.

Nach unserer Methode isolierte Parenchymzellen gefasteter Tiere konnten aus verschiedenen Substraten Glykogen synthetisieren (Abb. 2). Der Basiswert des Glykogengehaltès betrug $130 \pm 9,1 \mu \mathrm{g}$ Glykogen/g Feuchtgewicht. Aus Lactat bzw. Alanin in Konzentrationen von 10 mmol/1 wurden 48 bzw. $66 \mu \mathrm{g}$ Glykogen $/ 90 \mathrm{~min} \cdot \mathrm{g}$ Feuchtgewicht gebildet. Fructose in gleicher Konzentration ließ den Glykogengehalt der Zellen sogar um $430 \mu \mathrm{g} /$ $90 \mathrm{~min} \cdot \mathrm{g}$ Feuchtgewicht steigen. Aus Glucose in einer Konzentration von $30 \mathrm{mmol} / \mathrm{l}$ wurden im gleichen Zeit- 

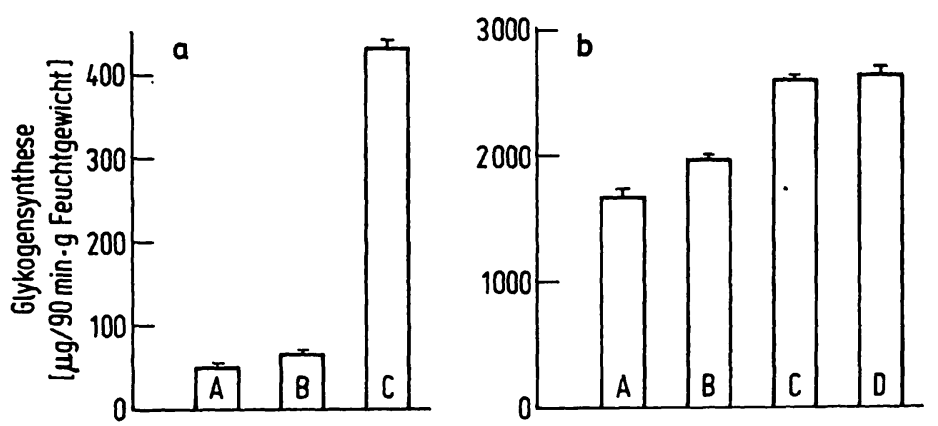

Abb. 2. Glykogensynthese isolierter Leberparenchymzellen von Hungertieren

$100 \mathrm{mg}$ Zellen wurden in $3 \mathrm{ml}$ Krebs-Ringer-Bicarbonat-Puffer $90 \mathrm{~min}$ inkubiert. Folgende Substrate waren dem Medium zugesetzt:
a) A: Lactat $10 \mathrm{mmol} / \mathrm{l} . \quad(\mathrm{n}=4)$
B: Alanin $10 \mathrm{mmol} / 1 \quad(n=6)$
C: Fructose $10 \mathrm{mmol} / 1 \quad(n=3)$
b) A: Glucose $30 \mathrm{mmol} / 1 \quad(n=5)$
B: Glucose $30 \mathrm{mmol} / 1$ und Alanin $5 \mathrm{mmol} / 1 \quad(n=5)$
C: Glucose $30 \mathrm{mmol} / 1$ und Alanin $10 \mathrm{mmol} / 1(n=5)$
D: Glucose $30 \mathrm{mmol} / 1$ und Alanin $15 \mathrm{mmol} / \mathrm{l}(\mathrm{n}=5)$

raum 1,65 $\pm 0,07 \mathrm{mg}$ Glykogen gebildet. Auf der Basis dieser Glucosekonzentration führten 5,10 oder $15 \mathrm{mmol} / \mathrm{l}$ Alanin zu signifikant $(\mathrm{p}<0,05)$ höheren Glykogensyntheseraten $(1,95 \pm 0,03 ; 2,61 \pm 0,03 ; 2,64 \pm 0,06 \mathrm{mg}$ Glykogen/90 min - g Feuchtgewicht). Der Effekt der Substratkombinationen ist deutlich höher als die Summe der Einzeleffekte.

Die Hormonsensibilität der nach unserer Methode isolierten Zellen wurde durch die Effekte von Glukagon, Adrenalin bzw. Insulin auf den Glykogenstoffwechsel und die Harnstoffsynthese belegt.

Der Einfluß von Glukagon und Adrenalin auf die Glykogenakkumulation (siehe Methodik) wurde in Parenchymzellen gefasteter Ratten bestimmt. Im Vergleich zu den Kontrollen, deren Glykogengehalt am Ende der Inkubation $(1,95 \pm 0,03 \mathrm{mg} / \mathrm{g}$ Feuchtgewicht) gleich $100 \%$ gesetzt wurde, betrug der Anstieg des Glykogens in Anwesenheit von Glukagon $(0,3 \mu \mathrm{mol} / 1)$ nur noch $15,1 \pm 1,6 \%$ $(n=5)$. Adrenalin in einer Konzentration von $1 \mu \mathrm{mol} / 1$ unterdrückte die Glykogenakkumulation fast vollständig $(4,1 \pm 0,7 \% ; n=5)$.

Beide Hormone stimulierten die Glykogenolyse in Parenchymzellen gefütterter Ratten. Der basale Glykogengehalt betrug $731 \pm 18,3 \mu \mathrm{g} / \mathrm{g}$ Feuchtgewicht. Der niedrige Ausganswert ist darauf zurückzufuihren, daß alle Schritte der Zellisolierung in glucosefreien Medien durchgeführt wurden, um so den Sauerstoffbedarf der Zellen während der kritischen Isolationsphase herabzusetzen. Eine 30-minütige Inkubation der Zellen führte zu einer Abnahme des Glykogengehaltes um 175,5 $\pm 14,4$ $\mu \mathrm{g} / \mathrm{g}$ Feuchtgewicht $(\mathrm{n}=5)$, während Glukagon $(0,3$ $\mu \mathrm{mol} / \mathrm{l})$ oder Adrenalin $(1 \mu \mathrm{mol} / \mathrm{l})$ den Glykogengehalt um $336,8 \pm 14,7$ bzw. $353,2 \pm 8,0 \mu \mathrm{g} / \mathrm{g}$ Feuchtgewicht senkten (jeweils $n=5$ ). Das entspricht einer Stimulierung der Glykogenolyse um 92,0 bzw. 101,4\%.

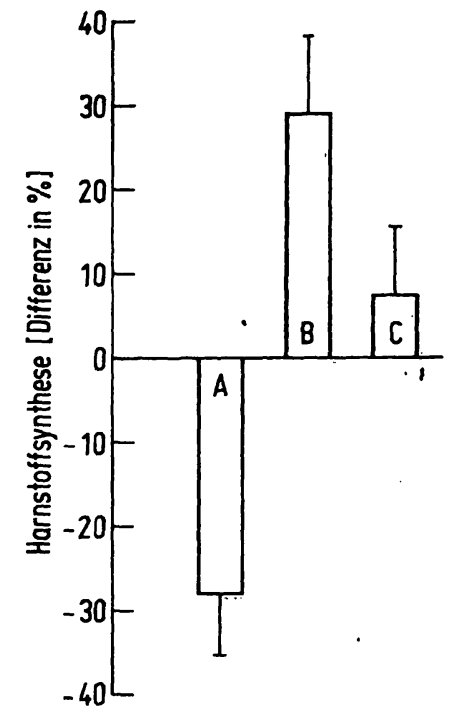

Abb. 3. Harnstoffsynthese isolierter Leberparenchymzellen $70 \mathrm{mg}$ Zellen wurden in $2 \mathrm{ml}$ Krebs-Ringer-BicarbonatPuffer mit $10 \mathrm{mmol} / 1$ Alanin $60 \mathrm{~min}$ inkubiert. Dem Medium waren folgende Hormone zugesetzt:
A: Insulin $10 \mathrm{nmol} / 1 \quad(\mathrm{n}=5)$
B: Glukagon $1 \mu \mathrm{mol} / 1 \quad(n=5)$
C: Insulin $10 \mathrm{nmol} / 1$ und Glukagon $1 \mu \mathrm{mol} / 1(\mathrm{n}=5)$
Angabe der durch die Hormone bewirkten Differenz. Ausgangswert: $4,10 \pm 0,35 \mathrm{mg} / \mathrm{h} \cdot \mathrm{g}$ Feuchtgewicht.

Abbildung 3 zeigt den Einfluß von Glukagon und Insulin auf die Harnstoffbildung isolierter Leberparenchymzellen. In Gegenwart von $10 \mathrm{mmol} / 1$ Alanin wurden 4,10 $\pm 0,35 \mathrm{mg}$ Harnstoff $/ \mathrm{h} \cdot \mathrm{g}$ Feuch tgewicht gebildet. Glukagon $(1 \mu \mathrm{mol} / \mathrm{l})$ steigerte die Harnstoffsynthese auf 5,31 $\pm 0,40 \mathrm{mg}$ (jeweils $\mathrm{n}=5$ ); das entspricht einer Stimulation um 29,5\%. Unter dem Einfluß von Insulin $(10 \mathrm{nmol} / \mathrm{l})$ wurden $2,95 \pm 0,31 \mathrm{mg}$ Harnstoff $/ \mathrm{h} \cdot \mathrm{g}$ Feuchtgewicht $(\mathrm{n}=5)$ gebildet, die Harnstofffreisetzung also um $28,0 \%$ vermindert. Die gleichzeitige Gabe beider Hormone in den angegebenen Konzentrationen führte zu einer Harnstoffproduktion von 4,40 $\pm 0,34$ $\mathrm{mg} / \mathrm{h} \cdot \mathrm{g}$ Feuchtgewicht $(\mathrm{n}=5)$, die sich nicht mehr signifikant vom Kontrollniveau unterschied.

\section{Diskussion}

Mit der von uns beschriebenen Isolierungsmethode lassen sich in nur 50 Minuten (Zeiten für Tieroperation und Zentrifugation eingerechnet) Leberparenchymzellen gewinnen. Den gleichen Zeitaufwand erfordern die Methoden von Berry (1) und Ingebretsen \& Wagle (8). Für die Verfahren von Howard (4), Hommes et al. (9), Johnson et al. (10) und Capuzzi et al. (11) müssen dagegen 75-90 Minuten veranschlagt werden.

Mit unserem Verfahren und der Methode nach Berry (1) lassen sich aus einer $10 \mathrm{~g}$ schweren Leber 4-6 $\times 10^{8}$ Zellen isolieren. Vergleichbar hohe Zellausbeuten wurden zwar von Ingebretsen (8) und Johnson (10) berichtet, ließen sich aber bei Anwendung ihrer Methoden von uns nicht reproduzieren (12). Hommes (9) und 
Capuzzi (11) erzielten mit einer mittleren Zellzahl von 3 bzw. $2 \times 10^{8}$ pro $10 \mathrm{~g}$ Leber eine geringere Ausbeute. Die Anzahl der von Howard (4) aus $10 \mathrm{~g}$ Leber isolierten Zellen betrug nach Angaben des Autors in Ubereinstimmung mit unseren Erfahrungen nur 2-8 $\times 10^{7}$ Zellen. Die Verfahren von Berry (1) und Howard (4) erfordern wie die Methoden von Capuzzi (11), Johnson (10) und Seglen (13) Kollagenase und Hyaluronidase. Howard (4), Berry (1) und Capuzzi (11) benutzen hohe Konzentrationen an Kollagenase (500-730 mg/1). Johnson (10) hat in ihrer Isolierungsmethode vor allem die Kollagenasekonzentration $(100 \mathrm{mg} / \mathrm{l})$ reduziert, um störende Einflüsse von Verunreinigungen der verwendeten Enzyme zu vermindern. Da andererseits jedoch eine Senkung der Kollagenasekonzentration eine Verlänges rung der Perfusionsdauer bedingt, benutzen wir eine mittlere Konzentration von $200 \mathrm{mg} / \mathrm{l}$. Auf Hyaluronidase konnten wir wie Ingebretsen (8) verzichten. Nach Angaben von Suzangar \& Dickson (14) führte die Verwendung von Hyaluronidase zu fast vollständigem Verlust zytoplasmatischer Enzyme wie z.B. Lactatdehydrogenase. Darüber hinaus beobachteten wir bei Isolierungsmethoden, in denen das Perfusat neben Kollagenase auch Hyaluronidase enthielt $(1,10)$, Rupturen der Pfortader durch Gefäßwanddigestion in der zweiten Hälfte der Perfusion. Solche Zwischenfälle beeinträchtigten die Reproduzierbarkeit der Methoden. Von Johnson (10) wurden z.B. Zellausbeuten von $2-10 \times 10^{8}$ Zellen pro Leber angegeben.

Hommes (9) konnte Leberzellen unter Verwendung von EDTA und Lysozym isolieren. Mit diesem Verfahren erhielten wir kaum Einzelzellen, sondern zumeist grössere Zellverbände. Da außerdem mit dieser Methode isolierte Zellen eine zerstörte Ultrastruktur, niedrige Atmungsaktivität und hohe Enzymverluste aufwiesen (15), dürfte das Verfahren keine brauchbare Alternative zu den Verfahren mit Kollagenase und Hyaluronidase darstellen.

Die Entfernung von Calciumionen ist ein entscheidender Schritt zur Zellisolierung. Bei einigen enzymatischen Verfahren wird neben der Verwendung von calciumfreien Puffern noch der Zusatz von calciumbindenden Substanzen wie Citrat $(11)$ bzw. EDTA $(1,9)$ empfohlen. Da z.B. EDTA zu Veränderungen der Permeabilität der Zellmembran und zur Zerstörung der Ultrastruktur von Zellen führte (11), verzichteten wir bei unserer Methode auf den Zusatz von calciumbindenden Substanzen. In Ubereinstimmung mit Seglen (13) stellten wir fest, daß bei hohen Durchflußratèn die Perfusion mit calciumfreien Puffern zum Auswaschen der Calciumionen ausreichend ist. An diesen Schritt schließt sich die enzymatische Phase der Isolierungsmethode mit Zugabe von Kollagenase und Calciumchlorid an. Calciumionen aktivieren die Kollagenase (16) und reduzieren den Kaliumverlust der Zellèn (4). Außerdem zeigten Zellen, die aus Leberschnitten in Anwesenheit von Calcium gewonnen wurden, höhere Sauerstoffaufnahmeraten (4) und eine besser erhaltene mitochondriale Struktur als Zellen, die in Abwesenheit von Calcium gewonnen wurden (17).

Im Rasterelektronenmikroskop ließen nach unserer Methode isolierte Parenchymzellen keine Substanzdefekte der Zellmembran erkennen (Abb. 1). Nur vereinzelt fanden sich Zellen, die als Zeichen der Membranschädigung unstrukturierte Vesikulationen aufwiesen.

In bezug auf Gluconeogenese und Proteinsynthese waren nach unserer Methode isolierte Parenchymzellen den Leberschnitten sowie den nach Howard (4) und Berry (1) isolierten Zellen deutlich überlegen. Die niedrige Syntheserate der Schnitte und der aus Schnitten isolierten Parenchymzellen (4) könnte darauf beruhen, daß die oberflächlich gelegenen Zellen durch den Schneidevorgang beschädigt werden und die Diffusion von Sauerstoff und Substraten in die tieferen Zellagen limitiert ist.

Die ATP/ADP- und Lactat/Pyruvat-Quotienten lagen bei den von uns isolierten Zellen im physiologischen Bereich.

Im Gegensatz zu den von Berry isolierten Parenchymzellen, die kein Glykogen synthetisieren konnten (1), bauten die nach unserer Methode isolierten Zellen aus verschiedenen Substraten Glykogen auf (Abb. 2). Vergleichbare Syntheseleistungen wurden von Wagle et al. (18) und Seglen (19) berichtet.

Die Hormonempfindlichkeit der von uns isolierten Zellen wurde durch die Wirkung von Glukagon und Adrenalin auf den Glykogenstoffwechsel von Parenchymzellen gefütterter und gefasteter Ratten belegt sowie durch den Nachweis des Insulin-Glukagon-Antagonismus bei der Harnstoffsynthese (Abb. 3). Diese Ergebnisse sind von besonderer Bedeutung, da die Hormonrezeptoren auf der äußeren Zellmembran enzymatischen Schädigungen wie z.B. durch Trypsin (10) leicht zugänglich sind. Die Integrität der Hormonrezeptoren kann außerdem als Bestätigung für den bei der morphologischen Analyse gewonnenen Eindruck einer intakten Zelloberfläche gewertet werden (20).

Die erzielten Ergebnisse und die dargelegten Vorteile gegenüber der Leberperfusion lassen nach der beschriebenen Methode isolierte Leberparenchymzellen als geeignetes Modell für die klinische Forschung erscheinen.

\section{Danksagung}

Diese Arbeit wurde im Rahmen des SFB 113 Diabetologie an der Universität Düsscldorf durch Sachmittcl gefördert.

Frau $M$. Störmer danken wir für gewissenhaftc Mitarbeit. 


\section{Literatur}

1. Berry, M. N. \& Friend, D. S. (1969), J. Cell Biol. 43, 506-520.

2. Morgan, J. F., Morton, H. J. \& Parker, R. C. (1950), Proc. Soc. Exp. Biol. Med. 73, 1-8.

3. Stadie, W. C. \& Riggs, B. C. (1944), J. Biol. Chem. 154, 687-690.

4. Howard, R. B., Lee, J. C. \& Pesch, L. A. (1973), J. Cell . Biol. 57, 642-658.

5. Lamprecht, W. \& Trautschold, J. (1970), in Methoden der enzymatischen Analyse (Bergmeyer, H. U., Hrsg.) 2. Aufl., Bd. 2, 2024-2032. Verlag Chemie, Weinheim/Bergstraße.

6. Seifter, R., Dayton, S., Novic, E. \& Muntwyler, E. (1950), Arch. Biochem. Biophys. 25, 191-200.

7. Rosenbauer, K. A. \& Schlösser, H. W. (1973), G-I-T Fachz. Lab. 17, 1370-137.3.

8. Ingebretsen, W. R. jr. \& Wagle, S. R. (1972), Biochem. Biophys. Res. Comm. 47, 403-410.

9. Hommes, F. A., Draisma, M. I. \& Molenaar, J. (1970), Biochim. Biophys. Acta 222, 361-371.

10. Johnson, M. E. M., Das, N.M., Butcher, F. R. \& Fain, J. N. (1972), J. Biol. Chem. 247, 3229-3235.
11. Capuzzi, D. M., Rothman, V. \& Margolis, S. (1971), Biochem. Biophys. Res. Comm. 45, 421-429.

12. Bojar, H. \& Reiners, K. (unveröffentlichte Ergebnisse).

13. Seglen, P.O. (1972), Exp. Cell Res. 74, 450-454.

14. Suzangar, M. \& Dickson, J. A. (1970), Exp. Cell Res. 63, 353-364.

15. Müller, M., Schreiber, M., Kartenbeck, J. \& Schreiber, G. (1972), Cancer Res. 32, 2568-2576.

16. Seifter, S. \& Harper, E. (1970), in Methods in Enzymology (Colowick, S. P. \& Kaplan, N. O., Eds.) Vol. XIX, 631, Academic Press, New York and London.

17. Howard, R. B. \& Pesch, L. A. (1968), J. Biol. Chem. 243, 3103-3109.

18. Wagle, S. R., Ingebretsen, W. R. jr. \& Sampson, L. (1973), Biochem. Biophys. Res. Comm. 53, 937-943.

19. Seglen, P.O. (1973), FEBS Letters $30,25-28$.

20. Wagle, S. R. \& Ingebretsen, W. R. jr. (1973), Biochem. Biophys. Res. Comm. 52, 125-129.

Dr. Hans Bojar

Institut für Physiologische Chemie II der Universität Düsseldorf

4000 Düsseldorf 